\section{Tel Amiel}

Universidade de Brasília, UnB

E-mail: tel@amiel.info

(D) https://orcid.org/0000-0002-1775-1148

\section{Thiago Pezzo}

Iniciativa Educação Aberta, IEA

E-mail:pezzo@protonmail.com

(iD https://orcid.org/0000-0001-6928-0862

Leonardo Ribeiro da Cruz

Universidade Federal do Pará, UFPA

E-mail: leocruz@ufpa.br

(iD) https://orcid.org/0000-0002-0383-4460

\section{Luisa Antunes Oliveira}

Universidade de Brasília, UnB

E-mail: luisabye@gmail.com

(D) https://orcid.org/0000-0001-9190-4950

Recebido em: 08/04/2021

Aprovado em: 25/08/2021

\section{Os modos de adesão e a abrangência do capitalismo de vigilância na educação brasileira}

\author{
Tel Amiel \\ Thiago Pezzo \\ Leonardo Ribeiro da Cruz \\ Luisa Antunes Oliveira
}

\section{Resumo}

É crescente a presença de plataformas e serviços de empresas privadas de software no ensino público. Isso traz à tona preocupações que incluem a autonomia tecnológica de redes de ensino e instituições, o tratamento de dados estratégicos e sensíveis e a privacidade de alunos, professores, gestores e responsáveis. No entanto, ainda pouco sabemos sobre a extensão dessas parcerias e o teor desses acordos. Este trabalho apresenta dados acerca da adesão de Instituições Públicas de Ensino Superior e Secretarias Estaduais de Educação brasileiras aos serviços educacionais de duas empresas associadas ao chamado 'capitalismo de vigilância', Google e Microsoft. Para tanto, foram utilizadas informações obtidas por meio de solicitações via Lei de Acesso à Informação. Os resultados indicam adesão massiva a serviços em 'nuvem' das empresas e pouca clareza quanto aos modos de adesão a essas parcerias. Por fim, os dados permitiram a validação e o aprimoramento de um script que faz a coleta automática de dados e permite uma visualização abrangente e atualizada do capitalismo de vigilância na educação brasileira.

Palavras-chave: Ensino público. Economia da informação. Tecnologia educacional. 


\begin{abstract}
Keywords: Public education. Information economy. Educational technology.

The modes of adoption and the pervasiveness of surveillance capitalism in Brazilian education

Public education has seen a surge in the presence of platforms from large private software companies. This in turn has raised concerns in regards to technological autonomy of school systems and institutions, the treatment of strategic and sensitive data, and the privacy of students, teachers, administrators, and legal guardians. However, we know little about the extent of these partnerships and the substance of these agreements. This article presents data on partnerships established between higher education institutions and state educational systems with two companies associated with 'surveillance capitalism': Google and Microsoft. The work is based on requests made via the Access to Information Law (LAI). The results indicate massive adherence to cloud services offered by these businesses and little clarity in regards to the content of the partnerships. Finally, the data allowed for the validation and improvement of a script that automatically collects data and allows for an up-to-date visualization of surveillance capitalism in Brazilian education.
\end{abstract}

\section{Resumen}

\section{Palabras clave:}

Educación pública. Economía de la información. Tecnología educativa.

\section{Los modos de adhesión y la difusión del capitalismo de vigilancia en la educación brasileña}

La presencia de plataformas y servicios de empresas privadas de software en la educación pública es cada vez mayor. Esto plantea preocupaciones que incluyen la autonomía tecnológica de las redes de enseñanza e instituciones, el tratamiento de datos estratégicos y sensibles, y la privacidad de los estudiantes, profesores, administradores y tutores. Todavía sabemos poco sobre el alcance de estas asociaciones y el contenido de estos acuerdos. Este trabajo presenta datos sobre la adhesión de las instituciones públicas de educación superior brasileñas y los departamentos de educación estatales a los servicios educativos de dos empresas asociadas al llamado "Capitalismo de Vigilancia", Google y Microsoft. El trabajo hizo uso de informaciones obtenidas a través de solicitudes mediante la Ley de Acceso a la Información de Brasil (LAI). Los resultados indican una adhesión masiva a los servicios "en la nube" de las empresas y poca información acerca de los modos de adhesión a los servicios. Por último, los datos permitieron validar y mejorar un script que recoge automáticamente los datos y permite tener una visión mas completa y actualizada del capitalismo de vigilancia en la educación brasileña.. 


\section{Introdução}

Temos acompanhado, nos últimos anos, o crescimento de relações entre as instituições públicas de ensino e grandes empresas de tecnologia envolvendo a utilização de serviços digitais para a educação. Corporações como Google e Microsoft, que figuram entre as mais valiosas empresas do mundo, têm oferecido às instituições de ensino do Brasil, em grande parte sem contrapartida financeira, acesso às suas tecnologias educacionais e espaço de armazenamento de informações em seus datacenters. Essas relações têm se intensificado, principalmente, desde a urgência da pandemia de Covid-19, dado o aumento da demanda por ensino remoto e a falta de estrutura própria para suportá-la.

No Brasil, a maior parte dos rendimentos das empresas envolvidas nesses acordos - em especial da empresa Google, que, como veremos, detém a maioria desse mercado - advém de um modelo de negócio assente na coleta e no tratamento de dados e na comercialização de perfis para o marketing comportamental. Hoje hegemônico na Internet, esse modelo de negócio se baseia na possibilidade de predição da ação dos indivíduos conectados por meio do rastreamento de seus comportamentos na rede.

Zuboff (2020, p. 80) denomina esse modelo de negócio, que tem a Google como pioneira, como Capitalismo de Vigilância: um novo modo de produção que extrai valor a partir de operações técnicas de coleta e análise de dados referentes a nossa sociabilidade digitalizada. Ele se baseia na ampliação das possibilidades de coleta de uma grande quantidade de dados de comportamentos dos usuários na rede. Esses dados, matéria-prima para os processos de produção dos 'produtos de predição', são capturados na utilização de serviços, plataformas ou dispositivos digitais ofertados como espaços para a nossa sociabilidade digitalizada. Eles podem ser tanto aqueles cedidos pelos usuários - como uma mensagem enviada a alguém, uma foto publicada em uma rede social, ou a busca por algum tema de interesse - como também podem ser metadados automaticamente coletados através de nossa interação com os serviços: que horas mandamos a mensagem e para quem, onde nos localizávamos quando tiramos uma foto pelo smartphone, a que horas entramos e saímos em um determinado serviço, dentre outros. Quanto mais áreas do nosso cotidiano forem digitalizadas e estiverem disponíveis em seus serviços, como no caso das relações educacionais, mais valor elas poderão extrair de seus investimentos (SILVEIRA; AVELINO; SOUZA, 2016).

As empresas do Capitalismo de Vigilância se valorizam, portanto, a partir da crescente e cumulativa digitalização do nosso cotidiano, feita, muitas vezes, sem o entendimento do valor agregado de milhares de pontos de dados de milhões de usuários - dados cruzados, vendidos e processados de formas que não temos como prever quando usamos serviços e aplicativos (SOLOVE, 2007; ETZIONI, 2012). É uma imensidão de dados que cresce exponencialmente, em quantidade e diversidade, à medida em que aumenta a base de usuários e/ou os tipos de uso desses serviços. 
Progressivamente, nossas relações de sociabilidade, de cidadania e de consumo estão sendo transferidas para o ambiente digital. Segundo o sociólogo Laymert Garcia dos Santos (2003), a transformação acelerada da nossa realidade em processos de codificação faz parte do que ele chama de "Virada Cibernética": um avanço da racionalidade instrumental, apoiada no cálculo matemático, na noção cibernética de informação, na capacidade de processamento e transmissão de dados das tecnologias digitais e na expansão dos mercados liberais. Esses processos resultaram no grande impulso econômico, técnico e científico da expansão da Internet e dos dispositivos digitais e na permeabilidade das chamadas 'novas tecnologias' em nossa vida cotidiana.

Essa digitalização é resultado não só dos próprios desdobramentos tecnológicos, mas também de processos políticos e econômicos que fazem parte do desenvolvimento do capitalismo contemporâneo (SANTOS, 2003; MOROZOV, 2018; FUCHS, 2020). Hoje, as tecnologias digitais das grandes empresas do Capitalismo de Vigilância são oferecidas como solução para problemas econômicos, políticos e sociais, aquilo que Evgeny Morozov (2013) denominou de Solucionismo Tecnológico.

No caso da educação pública brasileira, as tecnologias se apresentam, em parte, como solução para a incapacidade econômica das instituições públicas de ensino em gerenciar seu próprio parque tecnológico, que, por sua vez, é resultado direto das políticas de austeridade e da falta de investimento público em educação (PARRA et al., 2018; CRUZ; VENTURINI, 2020). No mesmo sentido, Maria Lindh e Jan Nolin (2016), ao analisar a entrada das tecnologias educacionais da Google na educação pública da Suécia, afirmam que o avanço das empresas de dados sobre os serviços prestados pelo Estado deve ser compreendido como o resultado de um esforço de décadas de transferência de bens e serviços públicos ao setor privado, visando a uma redução contínua dos gastos sociais. Sem investimento em soluções tecnológicas públicas e sem capacidade de gerenciar seus próprios processos digitais - cada vez mais necessários e numerosos -, as instituições públicas não teriam outro recurso à disposição que não as ferramentas e os serviços privados; assim, cedem parte de sua gestão de comunicação digital às grandes empresas de dados.

Ao contextualizar a rápida expansão do Capitalismo de Vigilância nos processos já existentes do capitalismo global, em especial os fluxos de trabalho, conhecimento e excedente econômico, podemos questionar como esse modelo de negócio atua sobre as históricas relações desiguais entre as diversas economias nacionais. É o que fazem Nick Couldry e Ulisses Mejias (2019), ao associarem a forma de atuação das grandes empresas de dados aos procedimentos históricos colonialistas de extração de valor em economias situadas na periferia do capitalismo mundial, caracterizando o que eles denominam de “Colonialismo Digital”. Os pesquisadores Rafael Evangelista e Rodrigo Firmino também orientam sua teorização sobre o conceito de Capitalismo de Vigilância de Zuboff para as possibilidades de compreensão de sua atuação em relação aos fluxos desiguais de conhecimento e de excedente econômico próprios do 
mercado internacional, em especial aqueles envolvendo as ricas economias do Norte - sede das grandes empresas de dados - e as economias dependentes do Sul Global (EVANGELISTA, 2019; EVANGELISTA; FIRMINO, 2020).

Entretanto, embora estejamos inserindo cada vez mais partes do nosso cotidiano nas redes do Capitalismo de Vigilância, pouco sabemos de sua atuação como mercado e do nosso papel nele. Temos pouca informação sobre seu funcionamento e suas tecnologias de coleta e análise de dados - baseadas em algoritmos e em aprendizado de máquinas - já que estão protegidas por propriedade intelectual e regras de segredo de negócios (COUTINHO; KIRA, 2019; CRUZ; SARAIVA; AMIEL, 2019). Como afirma Zuboff (2020), a privacidade e a transparência são direitos exercidos de forma muito desigual no Capitalismo de Vigilância. Enquanto se baseia na ampla coleta e tratamento de dados produzidos pelos usuários de suas plataformas, a ocultação de informações sobre sua atuação prejudica nossa compreensão sobre seu modelo de negócio e dificulta as possibilidades de regulação desse mercado. Essa desigualdade, no que diz respeito à transparência, é parte da sua atuação e fonte de acúmulo de capital, de conhecimento e de poder para essas empresas.

Nas Instituições Públicas de Ensino Superior (Ipes), dentre diversos problemas, a pouca transparência limita o debate sobre a tecnologia da informação, com consequências para a privacidade e a segurança das comunicações acadêmicas e científicas, além da necessidade de investimentos em tecnologias não voltadas ao vigilantismo. O mesmo vale para a educação básica, com o agravante do direcionamento para um público de crianças e adolescentes. Por fim, as decisões tomadas de forma desinformada acabam por realimentar o crescimento desse mercado.

A escassez de informações sobre a atuação do Capitalismo de Vigilância é ainda mais acentuada na educação. Estudos recentes em diversos países apontam para cenários preocupantes, alertando para a pouca compreensão, em escala global, acerca dos riscos desse mercado para a educação (ALIM et al., 2017; KWET, 2017).

Nesse estudo, temos como objetivo principal caracterizar o contexto brasileiro, apresentando dados relativos à adesão de Ipes e Redes Estaduais de Educação brasileiras aos serviços de empresas associadas ao Capitalismo de Vigilância. Coletamos dados por meio de solicitações formais (via Lei de Acesso à Informação - LAI) para identificar a adesão aos serviços educacionais em nuvem de duas empresas Google e Microsoft. Ademais, utilizamos os dados aqui apresentados para validar um método de coleta de dados automático (script) de computador (CRUZ; SARAIVA; AMIEL, 2019) que facilita o monitoramento dessas parcerias em grande escala. Finalizamos com uma análise dos modos de adesão (termos) a essas parcerias. Com isso, visamos contribuir com dados sobre o contexto e as possíveis ramificações dessas relações na educação básica e no ensino superior brasileiros. 
O projeto

O Projeto Educação Vigiada tem como objetivo mapear dados atuais sobre a relação de Ipes e Secretarias de Educação brasileiras com empresas associadas ao Capitalismo de Vigilância. Iniciou com estudos acerca da adesão realizada por duas grandes universidades aos serviços das empresas Google e Microsoft (PARRA et al., 2018) e atualmente segue com a busca por maior detalhamento acerca de como se dão essas parcerias, que são o objeto deste estudo.

Dados preliminares coletados por meio de solicitações em portais de transparência (LAIs), bem como as análises dos estudos de caso, indicaram-nos que a mudança dos serviços de e-mail das redes de ensino ou instituições para essas empresas poderia ser um bom indicativo de parcerias mais abrangentes. Seguindo essa lógica, o projeto criou um script, disponível em código aberto (CRUZ; SARAIVA; AMIEL, 2019), que é alimentado com dados de servidores de e-mails de instituições, posteriormente coletados e verificados de forma manual. O script busca e processa dados coletados por meio do comando host, que indica o servidor MX (para onde o servidor de nomes de domínio, ou Domain Name System - DNS, aponta o envio de e-mail), associado ao domínio específico (como, por exemplo, uel.br, para a Universidade de Londrina). Na página do Projeto, os dados são consolidados na forma de gráficos e de um mapa interativo, que permitem, com transparência, a apresentação dos dados coletados e de seus resultados agregados.

\section{Metodologia}

Neste trabalho, apresentamos os resultados da busca por informações sobre os acordos e parcerias realizados entre Ipes - aqui restritas a universidades e institutos federais -, Secretarias Estaduais de Educação e as duas empresas previamente mencionadas: Google e Microsoft. Os estados foram selecionados de forma aleatória, conforme o Quadro 1:

Quadro 1 - Instituições Públicas de Ensino Superior (Ipes) e Redes Estaduais de Ensino que compõem o estudo (continua)

\begin{tabular}{|c|l|}
\hline Estados (9) & \multicolumn{1}{c|}{ Ipes (30) } \\
\hline $\mathrm{CE}$ & $\begin{array}{l}\text { - Universidade Federal do Ceará (UFC); } \\
\text { - Instituto Federal de Educação, Ciência e Tecnologia do Ceará (IFCE). }\end{array}$ \\
\hline $\mathrm{GO}$ & $\begin{array}{l}\text { - Universidade Federal de Goiás (UFG); } \\
\text { - Instituto Federal de Goiás (IFG); } \\
\text { - Instituto Federal Goiano (IF-Goiano). }\end{array}$ \\
\hline $\mathrm{MG}$ & $\begin{array}{l}\text { - Universidade Federal de Minas Gerais (UFMG); } \\
\text { - Instituto Federal de Educação Ciência e Tecnologia de Minas Gerais (IFMG). }\end{array}$ \\
\hline PA & $\begin{array}{l}\text { - Universidade Federal do Oeste do Pará (Ufopa); } \\
\text { - Universidade Federal Rural da Amazônia (UFRA); } \\
\text { - Universidade Federal do Sul e Sudeste do Pará (Unifesspa); } \\
\text { - Universidade Federal do Pará (UFPA); }\end{array}$ \\
\hline
\end{tabular}




\begin{tabular}{|l|l|}
\hline & $\bullet$ Instituto Federal de Educação, Ciência e Tecnologia do Pará (IFPA). \\
\hline \multirow{3}{*}{ PE } & $\begin{array}{l}\text { - Universidade Federal Rural de Pernambuco (UFRPE); } \\
\text { - Iniversidade Federal de Pernambuco (UFPE); } \\
\text { - Instituto Federal de Pernambuco (IFPE); }\end{array}$ \\
\hline
\end{tabular}

Quadro 1 - Instituições Públicas de Ensino Superior (Ipes) e Redes Estaduais de Ensino que compõem o estudo (conclusão)

\begin{tabular}{|c|c|}
\hline Estados (09) & Ipes (30) \\
\hline RJ & $\begin{array}{l}\text { - Universidade Federal Fluminense (UFF); } \\
\text { - Universidade Federal do Rio de Janeiro (UFRJ); } \\
\text { - Universidade Federal do Estado do Rio de Janeiro (Unirio); } \\
\text { - Instituto Federal de Educação, Ciência e Tecnologia do Rio de Janeiro (IFRJ). }\end{array}$ \\
\hline RS & $\begin{array}{l}\text { - Universidade Federal de Santa Maria (UFSM); } \\
\text { - Universidade Federal do Rio Grande do Sul (UFRGS); } \\
\text { - Universidade Federal de Pelotas (UFPel); } \\
\text { - Instituto Federal do Rio Grande do Sul (IFRS). }\end{array}$ \\
\hline SE & $\begin{array}{l}\text { - Universidade Federal de Sergipe (UFS); } \\
\text { - Instituto Federal de Educação, Ciência e Tecnologia de Sergipe (IFSE). }\end{array}$ \\
\hline SP & $\begin{array}{l}\text { - Universidade Federal de São Paulo (Unifesp); } \\
\text { - Universidade Federal do ABC (UFABC); } \\
\text { - Universidade Federal de São Carlos (UFSCar); } \\
\text { - Instituto Federal de São Paulo (IFSP). }\end{array}$ \\
\hline
\end{tabular}

Fonte: elaborado pelos autores.

A equipe de pesquisa, composta por alunos de graduação, sob supervisão de dois docentes e um pesquisador associado, dividiu-se para solicitar informações por meio de três pedidos separados de acesso à informação nos portais de transparência do governo federal, no caso das Ipes, e nos portais dos estados, no caso das Secretarias de Educação, solicitando as seguintes questões:

1. Contratos específicos dos acordos pactuados, solicitando os documentos ou informações, com o seguinte teor: "Gostaria de solicitar todos os documentos ou, na falta desses, informações sobre acordos institucionais pactuados entre [a Instituição/o governo estadual, voltados para a Rede Estadual de Educação Básica] e as empresas Google e Microsoft nos últimos 10 anos".

2. Serviços específicos que foram alvo de termo de adesão entre a instituição e as empresas Google e Microsoft, com a pergunta: "Gostaria de saber quais serviços do Google e Microsoft foram objeto de termo de adesão [em nível institucional/pelo estado, voltados para a educação básica], incluindo, por exemplo, parcerias para uso de Google Apps for Education, Office 365 ou demais serviços e softwares oferecidos pelas referidas empresas". 
3. Responsável pelo serviço de hospedagem e armazenamento de e-mail institucional, com a pergunta: "Gostaria de saber quem (instituição ou empresa) armazena os e-mails do domínio institucional @XXX.XX”. E para os estados: "Gostaria de saber qual (instituição ou empresa) armazena os e-mails utilizados de forma institucional na Rede Estadual de Educação Básica".

De todas solicitações às instituições, somente uma foi negada, ${ }^{1}$ Das 27 solicitações enviadas aos estados, duas (Pará) não foram recebidas, mas posteriormente foram pedidos detalhes de contratos apontados na primeira resposta, e nove (de Goiás, Pernambuco e Sergipe) tiveram que ser refeitas no primeiro semestre de 2020, por problemas de acesso aos sistemas ou outras complicações.

Os dados foram tabulados e analisados de acordo com os seguintes critérios: primeiro, uma análise das respostas para entender se houve adesão aos serviços (a uma, nenhuma ou ambas as empresas). Segundo, buscamos analisar o modo de adesão, fosse no site das empresas, fosse por contrato específico, por meio de parceiros ou outro modelo. Todos os dados, na forma de um dataset, estão disponíveis com uma licença livre (AMIEL, et. al, 2021).

\section{Análise}

As parcerias levadas em consideração para o cálculo das porcentagens a seguir dizem respeito à adesão específica ao G Suite / Google Apps for Education (hoje denominado Google Workspace for Education), no caso da empresa Google, e ao Office 365 (hoje denominado Microsoft 365), no caso da Microsoft.

As respostas recebidas, muitas vezes, não eram diretas. Em alguns casos, não há uma confirmação clara da relação com as empresas, ou se as parcerias estão em vigor ou não. Nas análises abaixo, consideramos que houve adesão, portanto computamos positivamente qualquer indicação de contrato de larga escala para implementação dos sistemas nas redes estaduais e nas instituições. Computamos positivamente mesmo os casos em que havia indicação da data final do contrato, já que não podemos aferir se houve ou não renovação. Isso se justifica pela escala de transformação necessária para a adesão, tanto nas Redes Estaduais de Ensino quanto nas Ipes, que não é de fácil reversão. Por outro lado, não consideramos contratos de compras de licenças de softwares para serem utilizados em servidores ou instalados em computadores (por exemplo, um sistema operacional). Por fim, não computamos os casos em que houve indicação de parceira ou projeto de curto prazo com as empresas, sem indicação clara de adesão aos serviços supramencionados, já que, apesar de ser indicativo de uma futura parceria, não é insumo em grau suficiente de confiança para afirmar consequentes adesões.

Os quadros abaixo apresentam os dados consolidados por Redes Estaduais de Ensino (Quadro 2) e Ipes (Quadro 3). Os dados incluem o resultado da análise das respostas às perguntas 1 e 2, feitas via LAI 
(coluna Respostas LAI); depois, a reposta específica à pergunta 3, sobre o servidor de e-mail (coluna Servidor LAI); e, por fim, o resultado dos dados coletados via script (coluna Servidor script).

Os dados de LAI de 2019, devolvidos no período de novembro a dezembro de 2019, tiveram seus teores comparados com os resultados do script de 6 de dezembro de 2019. Os dados coletados via LAI em 2020 (somente os casos de alguns estados elencados abaixo), que tiveram retorno no período de junho a julho de 2020, foram comparados com dados de 20 de julho de 2020. Para efeitos de análise, havendo ao menos um domínio institucional atrelado à Google ou à Microsoft, definimos que a Secretaria ou a Ipes tiveram seus serviços associados às empresas.

\section{Resultados}

No que se refere às Secretarias de Educação, os documentos apontam que oito dos nove estados estabeleceram parcerias com pelo menos uma das empresas. São Paulo possui acordos com ambas as empresas; Rio de Janeiro e Goiás manifestaram a existência de acordos somente com a Microsoft; Ceará, Minas Gerais, Pará, Pernambuco e Rio Grande do Sul indicaram adesão aos serviços do Google; e, por fim, Sergipe não indicou adesão, somente um projeto pontual em parceria com escolas (Quadro 2, em destaque as inconsistências abordadas mais abaixo).

Quadro 2 - Secretarias Estaduais de Educação (continua)

\begin{tabular}{|c|c|c|c|c|}
\hline UF & Data da resposta & Respostas LAI & Servidor LAI & Servidor script \\
\hline CE & $\mathbf{1 1 / 2 0 2 0}$ & Google & Próprio & Próprio \\
\hline GO & $6-7 / 2020$ & Microsoft & Próprio & Microsoft \\
\hline MG & $11 / 2019$ & Google & Google & Google \\
\hline
\end{tabular}

Quadro 2 - Secretarias Estaduais de Educação (conclusão)

\begin{tabular}{|c|c|c|c|c|}
\hline UF & Data da resposta & Respostas LAI & Servidor LAI & Servidor script \\
\hline PA & $12 / 2019$ & Google & Google & Google \\
\hline PE & $7 / 2020$ & Google & Próprio & Próprio/Google \\
\hline RJ & $11 / 2019$ & Microsoft & Microsoft & Microsoft \\
\hline RS & $\mathbf{1 1 / 2 0 1 9}$ & Google & Próprio & Próprio \\
\hline SE & $\mathbf{7 / 2 0 2 0}$ & Google & Próprio & Próprio/Google \\
\hline SP & $\mathbf{1 2 / 2 0 1 9}$ & Google/Microsoft & Google/Microsoft & Microsoft \\
\hline
\end{tabular}

Fonte: elaborado pelos autores. 
Quanto ao estado do Rio Grande do Sul, o script corretamente identificou a característica institucional dos servidores, alinhado à resposta recebida via LAI que se referiu aos e-mails institucionais (seduc.rs.gov.br). Investigações futuras apontaram a existência de dois outros domínios (aluno.rs.gov.br e educacao.rs.gov.br) associados a servidores Google. Quanto ao estado de Pernambuco, a bifurcação se dá ao menos entre dois endereços: educacao.pe.gov.br (servidores próprios) e professor.educacao.pe.gov.br (servidores Google). O mesmo padrão se observou no Ceará, onde o servidor mapeado (crede01.seduc.ce.gov.br), utilizado no script, apontava para domínio próprio, mas a inconsistência nos levou à identificação de três novos domínios (aluno.ce.gov.br, prof.ce.gov.br, sistemas.seduc.ce.gov.br), todos associados à Google. Também se deu o mesmo em Sergipe, cujo domínio (seduc.se.gov.br) é próprio, mas os subdomínios aluno. e professor. são hospedados pela Google. Esta implementação é idêntica à utilizada em São Paulo, onde houve identificação correta de associação à Gafam, ${ }^{2}$ mas os públicos se dividem. O domínio originalmente identificado (educacao.sp.gov.br) está associado à Microsoft. Posteriormente, o domínio aluno.educacao.sp.gov.br foi identificado como pertencente à Microsoft, enquanto prof.educacao.sp.gov.br está associado à Google.

No caso de Goiás, o script corretamente identificou a parceria com a Google. No entanto, o terceiro pedido de LAI enviado, focado na questão dos servidores de e-mail, indicou que "[...] a Seduc [Secretaria de Educação] conta com servidores próprios e realiza toda a administração dos e-mails institucionais da Seduc-GO”. Note-se que, no primeiro pedido, há indicação de que o serviço poderia estar em vias de efetivação, quando se afirma que a Microsoft “[...] está liberando o Office 365 e o e-mail e o pacote Office educacional". Novo pedido de LAI, feito em outubro de 2020, confirmou a hospedagem de e-mails em servidores Microsoft.

Quadro 3 - Instituições Públicas de Ensino Superior (Ipes) (continua)

\begin{tabular}{|c|c|c|c|c|}
\hline UF & Ipes & Termos (LAI) & Servidor (LAI) & Servidor (script) \\
\hline CE & UFC & Google/Microsoft & Google & Google \\
\hline CE & IFCE & Google & Google & Google \\
\hline GO & UFG & Google & Google & Google \\
\hline GO & IFG & Google/Microsoft & Google & Google \\
\hline GO & IF-Goiano & Google & Erro na pergunta & Google \\
\hline MG & IFMG & Google/Microsoft & Próprio & Próprio \\
\hline MG & UFMG & Próprio & Próprio & Próprio \\
\hline PA & Ufopa & Próprio & Próprio & Próprio \\
\hline PA & UFRA & Próprio & \\
\hline
\end{tabular}




\begin{tabular}{|c|c|c|c|c|}
\hline PA & Unifesspa & Google/Microsoft & Próprio & Próprio \\
\hline PA & IFPA & Próprio & Próprio & Próprio \\
\hline PA & UFPA & Próprio & Próprio & Google \\
\hline PE & UFRPE & Microsoft & Próprio & Próprio \\
\hline PE & IFSPE & Google & Google & Google \\
\hline PE & IFPE & Google & Google & Google \\
\hline PE & UFPE & Google & Google e Próprio & Próprio \\
\hline RJ & UFF & Google & Não respondido & Próprio \\
\hline RJ & UFRJ & Google/Microsoft & Google & Google \\
\hline RJ & Unirio & Google & Google & Google \\
\hline RJ & IFRJ & Google & Google & Google \\
\hline RS & IFRS & Google & Google e Próprio & Google \\
\hline RS & UFSM & Google & & \\
\hline
\end{tabular}

Quadro 3 - Instituições Públicas de Ensino Superior (Ipes) (conclusão)

\begin{tabular}{|c|c|c|c|c|}
\hline UF & Ipes & Termos (LAI) & Servidor (LAI) & Servidor (script) \\
\hline RS & UFRGS & Próprio & Próprio & Próprio \\
\hline RS & UFPel & Próprio & Próprio & Próprio \\
\hline SE & IFS & Google/Microsoft & Microsoft & Microsoft \\
\hline SE & UFS & Google/Microsoft & Próprio & Próprio \\
\hline SP & IFSP & Google/Microsoft & Google e Próprio & Próprio \\
\hline SP & Unifesp & Google & Google & Google \\
\hline SP & UFABC & Microsoft & Microsoft & Próprio \\
\hline SP & UFSCar & Google/Microsoft & Google & Google \\
\hline
\end{tabular}

Fonte: elaborado pelos autores.

Os dados levantados na análise envolveram 30 Ipes, de nove estados (Quadro 3, inconsistências em destaque). Constatou-se que 23 instituições (77\%) informaram ter realizado parcerias com pelo menos uma das referidas empresas. Do total, 2 Ipes (7\%) apontaram parcerias apenas com a Microsoft; 12 (40\%) 
indicaram parcerias exclusivamente com o Google; e 9 (30\%) apontaram a existência de parcerias com ambas as empresas. Somente 7 (23\%) afirmaram não possuir acordos com nenhuma delas.

Das instituições que negaram acordos existentes, a UFPel manifestou a intenção de adesão. O IFPA, embora tenha negado possuir contratos com as empresas, confirmou uma adesão de serviços 'em andamento', por meio da Rede Nacional de Pesquisa (RNP), mas sem especificar quais os tipos de serviços, o que nos levou a classificar o Instituto como tendente à adesão. A UFSM, que confirmou acordo com a Google (há indicação de processo no site da instituição, porém no link não existem anexos), indicou estar viabilizando a ampliação dos serviços já prestados pela empresa.

As inconsistências observadas entre os pedidos via LAI e o script seguem os padrões já identificados nos estados. A Unifesspa indicou que, apesar da contratação dos serviços, não faz uso do e-mail das empresas; o mesmo foi relatado pela UFPA. Posteriores pedidos de LAI para ambas as instituições confirmam que houve migração dos serviços de e-mail para a Google. A UFRPE já fazia uso dos serviços da Microsoft, e novo pedido de LAI indicou que, posteriormente, a instituição aderiu à Google e migrou seus serviços de e-mail para essa empresa.

Em quatro casos, houve disparidade nos dados, por conta da existência de bifurcação de domínios, sendo um institucional, com administração própria, e outro para públicos específicos, apontando a relação com as empresas: no IFSP (ifsp.edu.br e aluno.ifsp.edu.br - Google); na UFABC (ufabc.br e aluno.ufabc.br - Microsoft); na UFF (uff.br e id.uff.br - Google); e, por fim, no caso da UFS, o domínio institucional (ufs.br) é próprio, mas a inclusão do domínio academico.ufs.br confirma a migração para a Google.

Uma excepcionalidade é o caso da UFRJ, que, apesar da associação com ambas as empresas, indica fazer somente uso de servidores institucionais para $e$-mail.

\section{Modo de adesão}

Neste momento da pesquisa, reflete-se acerca de quais modos formais de associação foram utilizados pelas secretarias educacionais e Ipes para se vincularem aos serviços da Microsoft e da Google. Das nove secretarias, somente três enviaram os documentos solicitados. São Paulo encaminhou os termos de convênio estabelecidos com ambas as empresas. Pernambuco enviou termo de cooperação técnica com a Google, e Sergipe enviou documento que trata de aparente projeto-piloto, já finalizado, não considerado para os propósitos desta pesquisa.

Sem anexo ou indicação de instrumento formalizado, as outras parcerias só puderam ser estimadas pelas palavras utilizadas. A análise, porém, é delicada, devido à variabilidade das terminologias:

Quadro 4 - Estados e modos de contratação (continua)

\begin{tabular}{|c|c|}
\hline Estado & Modo de contratação \\
\hline Ceará & Incerto \\
\hline
\end{tabular}




\begin{tabular}{|c|c|}
\hline Goiás & Incerto \\
\hline Minas Gerais & Convênio/Contrato não oneroso \\
\hline Pará & Contratação \\
\hline Pernambuco & Termo de cooperação técnica \\
\hline
\end{tabular}

Quadro 4 - Estados e modos de contratação (conclusão)

\begin{tabular}{|c|c|}
\hline Estado & Modo de contratação \\
\hline Rio de Janeiro & Termo de responsabilidade \\
\hline Rio Grande do Sul & Termo de cooperação \\
\hline São Paulo & Termo de convênio \\
\hline Sergipe & Acordo de cooperação (projeto) \\
\hline
\end{tabular}

Fonte: elaborado pelos autores.

A ausência de terminologia precisa fez com que os modos de contratação das Secretarias Estaduais de Educação de Ceará e Goiás fossem classificados como incertos. Ambas justificaram que os produtos e serviços eram gratuitos. Em alguns casos, houve intermediação de empresas (como no caso do contrato com a Google em São Paulo), e, no caso de Pernambuco, houve intermediação do Conselho de Secretários Estaduais de Educação (Consed).

A elucidação acerca do tipo de instrumento jurídico utilizado se torna um processo complexo. No caso de Pernambuco, o anexo é um Termo de Cooperação Técnica firmado entre a Google e o Consed. No entanto, na seção ‘Obrigação das Partes', o documento indica que cabe ao Consed

[...] disponibilizar as [sic] secretarias estaduais de educação, o uso da plataforma 'G Suite for Education’ em todas as unidades que compõem estrutura da secretaria de educação, tais como gabinetes, assessorias, coordenadorias, divisões, diretorias, supervisões e unidades educacionais.

Como o Termo foi enviado como resposta às duas primeiras perguntas, somos levados a entender que foi este o mecanismo utilizado para formalizar a adesão do estado aos serviços. O termo anexado, no entanto, parece ter outro objetivo. Inclui um Plano de Trabalho específico (chamado de 'Convênio'), que tem como um de seus objetivos "[...] difundir o uso institucionalizado dos aplicativos do Google entre os professores de educação básica" . Cabe à parceira (Consed) definir seis escolas públicas em três estados diferentes para execução do plano. Apresenta prazos e três metas específicas: a capacitação de 324 educadores para uso de Chromebooks e G Suite for Education, elaboração de atividades com alunos e elaboração de um relatório de avaliação pedagógica (do G Suite for Education) por parte dos professores.

Quanto às Ipes, houve novamente dificuldades para a identificação dos instrumentos e modalidades de parcerias entre instituições e empresas. Recorrentes foram as respostas sem anexo de documentação 
formal ou indicação de link para consulta. Outras vezes, a indicação de parceria não era mencionada, tendo que ser depreendida dos textos fornecidos. Dentre as respostas obtidas, 8 (27\%) afirmaram que o modo de adesão às empresas se deu pela via on-line, muitas vezes com a apresentação de termos de serviços padrão dos sítios das empresas. Do total, 2 respostas (7\%, IFSP, IFS) indicaram em seus documentos, com alguma clareza, a existência de um contrato. Por fim 4 (13\%, UFG, IFPA IFCE e Unirio) citaram a intermediação da Rede Nacional de Ensino e Pesquisa (RNP) como intermediários da adesão- Note-se o IFCE, que afirma que "[...] instituições públicas de ensino que utilizam qualquer solução da plataforma Microsoft o fazem pela RNP via plataforma [Comunidade Acadêmica Federada] CAFe, não tendo convênio direto com a Microsoft".

Também aqui, como nas respostas das Secretarias Estaduais de Educação, as adesões on-line parecem não considerar a importância do aceite de termos de serviço que, efetivamente, vinculam as partes ao cumprimento de determinadas regras legais, em nada adaptadas a realidades universitárias tão diversas, tampouco ao contexto nacional (LIMA, 2020). Parecem contradizer também (como sabemos por outros casos aqui relatados) a informação de que a adesão é feita de forma exclusiva via RNP.

Algumas poucas instituições enviaram documentos comprobatórios das decisões sobre a adesão às empresas por parte de comitês gestores, mas não os efetivos instrumentos de parceria, como o IFS, o IFSPE e a Unifesp. Os debates aqui apontam questões similares às que foram elencadas em estudos prévios, incluindo limitações orçamentárias e de 'qualidade' ou mesmo a possibilidade de expansão dos serviços institucionais existentes (PARRA et al., 2018; CRUZ; VENTURINI, 2020).

Chama a atenção o grande número de respostas em que não é possível identificar exatamente o modo de adesão das Ipes a essas grandes empresas de software. Além disso, a inexistência de vínculos institucionais formais não implica a ausência de relações entre Microsoft/Google e departamentos, grupos de pesquisa e laboratórios, tampouco de adesão individual por parte de professores, servidores e discentes - situação que é sugerida em diversas das respostas recebidas. Foi mais difícil mensurar a abrangência de serviços e produtos nas Ipes, o que indica que subdimensionamos o nível de adesão aos serviços ao analisar os dados em nível institucional.

\section{Aspectos legais}

Em muitos dos casos são relatados processos de adesão pelo próprio site da empresa. De fato, a adesão inicial ao G Suite for Education pode ser feita no próprio site, em formulário simples, inserindo-se os dados da instituição. O usuário imediatamente tem acesso ao painel e pode adicionar um número restrito de contas até que seja verificada a procedência do site pela Google. A resposta recebida pela UFC indica esse processo: 
A adesão se deu por preenchimento de formulário on-line à época de seu início e por comprovação da detenção de autoridade do domínio ufc.br. A assinatura gratuita do G Suite for Education aderido pela UFC em seus serviços principais é regida pelo Aviso de Privacidade do G Suite for Education (https://gsuite.google.com/intl/pt-BR/terms/ education_privacy.html) e pelo Contrato do $G$ Suite for Education (https://www.google.com/apps/intl/pt- BR/terms/education_terms.html). (ênfase nossa).

No que tange à relação com a Microsoft, em sua resposta, a UFC nos diz:

[...] informamos que não há nenhum contrato formal entre a Microsoft e a UFC. A empresa Microsoft disponibiliza para as Instituições de Ensino Superior (IES) o uso gratuito do Office 365 Online A1, não necessitando de assinatura de contrato. Para tanto, basta que alguém da instituição solicite o acesso via e-mail institucional (é feita uma verificação e confirmação pelo e-mail). (ênfase nossa).

Procedimento similar é relatado pela UFPRE :

A UFRPE realizou, nos últimos 10 anos, o cadastro do domínio institucional "ufrpe.br", no site da Microsoft para uso do Office 365 Educação on-line, no plano conhecido comercialmente como Office 365 Al, gratuito. O cadastro é feito on-line, onde a Microsoft analisa se o domínio fornecido (ex: ufrpe.br) é realmente de uma instituição de ensino e libera o serviço para todos os usuários que acessem através do e-mail institucional cadastrado. Não existem documentos formais de adesão institucional, nem de parcerias, tudo foi feito digitalmente sem registro físicos que possam ser apresentados. (ênfase nossa).

O mesmo procedimento foi adotado pela Unirio, que indica que o 'contrato’ é feito “[...] direto pelo site do Google for Education. Informo ainda que a solução é de custo zero”. Na mesma linha, o IF-Goiano indica que foi realizada “[...] a adesão on-line, portanto possuímos o termo de adesão padrão disponível no site da solução".

Há ainda, ao que parece, um desentendimento sobre a natureza da relação estabelecida entre as instituições e as empresas. O IFG, por exemplo, respondeu ao pedido de 'acordos institucionais' com uma negativa: o "Instituto Federal de Goiás não possui acordo pactuado com Google ou Microsoft.” No entanto, na pergunta seguinte, indica que “[...] utiliza os serviços Google For Education e também Microsoft Office 365, ambos fornecidos gratuitamente a Instituição". O mesmo teor pode ser visto na resposta dada pela UFPE , que pontua não ter “[...] nenhum instrumento jurídico celebrado entre a UFPE e as empresas”; no entanto, a segunda resposta revela a parceria: "Foi estabelecido acordo entre a UFPE e o Google para utilização do Google for Educational [sic]." Por fim, a UFSCar indica não ter 'parcerias', mas aponta, no mesmo documento, que "[...] parcerias foram estabelecidas por meio do aceite da instituição aos termos de serviços dos respectivos produtos".

Um caso à parte, o IFSE apresenta uma instrução normativa interna para orientação de uso do Google for Education e Office 365 Education, dentre outros serviços. Esclarece que (seguindo os termos das próprias empresas) o serviço é oferecido somente enquanto houver vínculo com a instituição e deixa 
claro que o uso dos serviços é educacional. Sugere que o aluno ou docente só poderá fazer uso da sua conta institucional ao acatar os termos de uso e os contratos das respectivas empresas (Google e Microsoft), não indicando um termo ou contrato específico assinado pelo Instituto. Aponta também que alunos menores de 18 anos devem ter consentimento assinado pelos pais ou responsáveis para fazer uso do serviço, através de um termo que pede concordância com as cláusulas dos produtos contratados e com a Lei Americana de Proteção da Privacidade On-line de Crianças de 1998 (Coppa):

$$
\begin{aligned}
& I \text { - Os termos do contrato "Google Apps for Education (online) } \\
& \text { "https://www.google.com/apps/intl/pt-BR/terms/education_terms.html”, } \\
& \text { II - Os termos da "Política de Uso Aceitável do Google Apps" } \\
& \text { "https://www.google.com/apps/intl/pt-BR/terms/use_policy.html” } \\
& \text { III - Os termos da Lei americana de Proteção da Privacidade On-line das Crianças de } 1998 \\
& \text { “https://www.ftc.gov/system/files/2012-31341.pdf”"(Original em inglês) } \\
& \text { “https://jus.com.br/artigos/23373/a-atualizacao-da-lei-americana-de-protecao-dos-dados-das- } \\
& \text { criancas-na-internet” (Versão em Português) } \\
& \text { IV - Os termos da "Assinatura do Microsoft Imagine" } \\
& \text { "https://catalog.imagine.microsoft.com/pt-br/about/LicenseAgreement" } \\
& V \text { - Os termos do "Contrato de Serviços da Microsoft" } \\
& \text { "https://www.microsoft.com/pt-br/servicesagreement". }
\end{aligned}
$$

Cabe ao responsável a leitura e compreensão de todos os termos acima, para então dar sua anuência.

Dos documentos recebidos, é esclarecedor o parecer da procuradoria do IFCE, contactada pelo Diretor de Gestão de Tecnologia da Informação, em consulta acerca da possibilidade e legalidade da adoção do Google for Work (à época, espécie de nome guarda-chuva dado ao Google Suite na educação e nas empresas) por parte da instituição. O parecer considera a manifestação dos solicitantes, que expressam insatisfação com o serviço de e-mail prestado pela SERPRO (Serviço Federal de Processamento de Dados) e o custo (mensal, de R\$ 11 mil) para manutenção do serviço de e-mail. A procuradoria ressalta a importância da 'economicidade' e 'eficiência' como princípios da administração, citando, sem ênfase, o interesse público e a moralidade do acordo.

Parece-nos que, excetuando-se alguns poucos casos, a facilidade de adesão institucional pode passar por cima de preocupações importantes quanto à privacidade, à coleta e ao tratamento de dados. A adesão aos serviços nos sites de empresas pode levar à errônea conclusão de que não há necessidade de 'assinatura de contrato' ou formalização, particularmente porque se trata de um serviço 'gratuito'. De fato, os termos e contratos padrões são aceitos pelas instituições, o que nos leva a questionar a existência de análise jurídica do seu conteúdo.

O IFCE não é a única instituição que apresenta preocupações com a legalidade da adesão aos serviços. Na resposta recebida da UFSCar, mencionam-se os padrões de segurança e as certificações de ambas as empresas, bem como se ressalta (antes da efetivação da Lei Geral de Proteção de Dados - LGPD) que, 
[...] embora não haja legislação vigente no Brasil especifica sobre a matéria de proteção de dados [...], os produtos adotados possuem conformidade com legislações de outros países, como a [General Data Protection Regulation] GPDR (Europa), [Family Educational Rights and Privacy Act] FERPA (EUA), [Personal Information Protection and Electronic Documents Act] PIPEDA (Canadá), o que denota o compromisso dos provedores com a privacidade dos dados dos usuários. (ênfase nossa).

Como a manifestação deixa claro, todas as legislações mencionadas acima, como a Coppa e a GDPR, não têm qualquer validade ou implicação para o Brasil. No entanto, entendemos que, ao apontarem termos de privacidade, conformidade com legislação e outros critérios de segurança, as instituições demonstram preocupação com esse tema.

Como evidenciamos em estudos anteriores (PARRA et al., 2018), nas instituições, há limitado engajamento, debate e discussão sobre a adesão a esses serviços. As discussões, que às vezes ocorrem em diretorias e conselhos, contam com limitada transparência e participação de poucos atores das instituições. Nas palavras de Borgmann (1993, p. 114): “[...] exaltar o consumidor é negar o cidadão. Quando os consumidores começam a agir, as decisões fundamentais já foram tomadas" ${ }^{4}$ Ou seja, restaria aos docentes, gestores e alunos, quando muito, o verniz de uma escolha solitária.

Isso nos leva a questionar a responsabilização do usuário final. Cabe ao docente ou ao aluno a decisão sobre a adesão. Cabe ao pai ou responsável a leitura de diversos termos de uso - sempre on-line (lembrando que muitos têm péssimo ou limitado acesso à Internet), por vezes em inglês. Cabe também a alunos, docentes e responsáveis compatibilizar as normas das empresas com os termos de uso (se houver) da própria instituição, para decidir de forma autônoma sua adesão ao serviço. Como diz Etzioni (2012, p. 941):

[...] a sugestão de que se as corporações simplesmente declarassem quais são seus padrões de privacidade, os consumidores poderiam escolher aqueles que lhes convêm, é errada, se não enganosa. Os termos são redigidos em linguagem legal complexa, com terminologia que poucos podem transpor; as configurações de privacidade fornecidas são complexas, confusas e frequentemente revisadas - depois que os usuários já postaram informações no site que não podem mais apagar ${ }^{5}$. (tradução nossa).

Existe também uma falsa noção de exclusividade de responsabilidade por parte das empresas no que tange à privacidade dos usuários. Os próprios termos das empresas deixam claro que cabe às instituições, por exemplo, garantir a anuência de pais e responsáveis quando o aluno tem menos de 18 anos. Ademais, nos termos da LGPD, são as instituições e redes que atuam como controladoras dos dados, ou seja, há corresponsabilidade entre as partes (LIMA, 2020). Cabe também à instituição definir ao menos alguns parâmetros de coleta de dados e administrar de forma geral o sistema, por meio de um painel de controle de serviços e aplicativos. 
Por último, como já ressaltado em outro trabalho (AMIEL, 2020), com o crescente uso de aplicativos e serviços dessas e de outras empresas associadas ao Capitalismo de Vigilância em atividades escolares (CETIC.BR, 2019), não aventamos a existência de formas, muitas vezes pouco sutis, de coerção em sala de aula ou nos locais de trabalho. Se um estado, um município ou uma instituição estabelece que o e-mail institucional (e não um outro subdomínio, inicialmente opcional) será gerenciado por uma empresa, que margem tem um docente ou gestor para não fazer uso dessa conta? Como é indicado em uma apresentação (não mais disponível no site) sobre o G Suite, criada pela Secretaria de Educação do Estado de Minas Gerais, o e-mail institucional é "meio oficial de comunicação", tem "identidade corporativa", "vale como documento oficial" e "pode ser auditado". Qual margem de manobra há para um docente da rede ou gestor público que não queira fazer uso desses sistemas?

O problema se intensifica quando analisado à luz da queda de investimento nos gastos públicos brasileiros (particularmente na educação pública) como saída para a situação de austeridade econômica. A contínua redução no orçamento produz consequências negativas na capacidade de gerenciamento das Tecnologias de Informação e Comunicação (TIC) das instituições e redes públicas de ensino. Com a falta de investimento para a manutenção e ampliação dos datacenters, bem como para a contratação de pessoal, as instituições educacionais se deparam com o crescimento da demanda por serviços digitais - como o armazenamento e gerenciamento de e-mail, de sites e de aplicativos - e com a impossibilidade de gerenciálos (PARRA et al., 2018). Desse modo, as oportunidades oferecidas pelas empresas privadas - contas de e-mail para toda a comunidade escolar, armazenamento amplo de dados e serviços de software para serem utilizados em sala de aula ou nos processos de pesquisa, muitas vezes sem contrapartida financeira para a instituição pública - passam a ser encaradas como um serviço necessário.

\section{Conclusão}

Com este trabalho, apresentamos dados novos sobre a preocupante e crescente presença de grandes empresas associadas ao Capitalismo de Vigilância na educação brasileira. Os dados obtidos oferecem informações que nos permitem identificar nuances nessas relações, incluindo: os atores que estão envolvidos, as formas de adesão, bem como algumas justificativas e processos adotados pelos entes estudados. A solicitação de dados através da Lei de Acesso à Informação parece ser um caminho lento, mas necessário para esse fim, dado que muitos processos e documentos se encontram fechados nas bases de dados das instituições e da administração pública, quando deveriam ser objeto de transparência ativa.

É preocupante a variedade de modelos de adesão aos serviços, que, com poucas exceções, parecem ser pouco específicos e apropriados. Por um lado, parece haver um equívoco de interpretação quanto à 'gratuidade' dos serviços e aos seus respectivos procedimentos. Como exemplo, para entes da administração pública federal, o Decreto $n^{\circ}$ 9.764, de 11 de abril de 2019, enumera uma série de passos 
para a efetivação da doação, que se inicia com “[...] a abertura, por meio de publicação de edital” (BRASIL, 2019, art. 8). A inexigibilidade de contrato, regida pelo Artigo 25 da Lei no 8.666, de 21 de junho de 1993 (BRASIL, 1993), só é aventada em casos de "inviabilidade de competição", que, se aplicada ao caso abordado neste trabalho, seria facilmente desconstruída, haja vista a presença de pelo menos duas empresas ofertando serviços muito similares.

Parece-nos que, independentemente das possibilidades legais, que estão fora do escopo deste trabalho, as instituições e redes deveriam no mínimo adotar processos públicos e transparentes para chamamento, análise de propostas e contratação de serviços. Ou seja, deveriam adotar outros critérios além da 'economicidade', incluindo os princípios de "moralidade, publicidade e eficiência" (BRASIL, 1988, art. 37), essenciais à administração pública.

Por fim, esta análise nos permitiu validar um script que varre de forma automática os servidores de e-mail de instituições e fornece um panorama sempre atualizado das relações entre Ipes, redes de ensino públicas e as referidas empresas. Os dados são atualizados regularmente no site do projeto Educação Vigiada e conferem um bom grau de confiabilidade às informações.

Como limitações, reconhecemos que o estudo trabalhou com um recorte que privilegiou Ipes e Redes Estaduais de Ensino. As conclusões e análises feitas aqui se restringem aos casos analisados. No entanto, a diversidade de instituições e redes analisadas, assim como a existência de padrões claros nas respostas, permite-nos um primeiro panorama confiável das Redes Estaduais de Ensino e Ipes federais brasileiras.

Algumas respostas à LAI tiveram que ser analisadas a partir de um registro discursivo. O objetivo foi apreender certas relações contratuais a partir dos termos utilizados nos textos das respostas, e não pelo exame de documentação legal, como era esperado. Isso porque a grande maioria das respostas não veio acompanhada de documentação oficial, nem indicou sua existência nos sites institucionais ou da administração pública. Em outros casos, os processos indicados não puderam ser encontrados nos sítios citados ou em buscas na web, portanto tiveram de ser novamente solicitados (e alguns o foram). Talvez o mais preocupante tenha sido as justificativas de ausência de protocolos de adesão e também os documentos, que, mesmo com suas localizações indicadas, de fato não estavam disponíveis. A análise dos procedimentos legais, portanto, deve ser alvo de estudo futuro.

O não envio da documentação é fruto, em grande parte, da adesão a serviços nos sites das próprias empresas; as instituições parecem não considerar os problemas desse modelo, que é feito sem 'contrato' e com aceitação de termos genéricos, muitas vezes não aplicáveis ao contexto brasileiro.

Em próximas etapas do estudo, sugerimos a possibilidade expandir a solicitação de informações para incluir todas as Ipes e Redes Estaduais de Ensino, a fim de obter uma análise completa desse fenômeno. Ademais, muito dos casos aqui apontados merecem um estudo mais aprofundado, com novas solicitações 
de documentação, entrevistas e outros mecanismos complementares de coleta de dados, para que possamos melhor entender como se dão as parcerias entre as empresas e nossas instituições de ensino. É importante também um estudo mais criterioso das diversas ramificações dessas parcerias, diante dos problemas suscitados pelo Capitalismo de Vigilância na educação, incluindo as consequências para a privacidade de dados de alunos, professores e gestores, processos de fidelização institucional e de usuários, criação de perfis, venda e comercialização de dados (anônimos ou não), dentre outros.

Em tempos de pandemia e aceleração do discurso em torno de uma educação 'híbrida', consideramos de extrema importância que atores da educação estejam cientes dos problemas aqui apontados e exijam transparência, participação e autonomia nos processos envolvendo sistemas tecnológicos na educação.

\section{Disponibilidade de dados}

Todo o conjunto de dados foi disponibilizado em repositório aberto (AMIEL, et. al, 2021). O script que ampara os resultados apresentados neste estudo foi disponibilizado no repositório GitLab e está disponível em https://gitlab.com/ccsl-ufpa.

\section{Notas}

A primeira solicitação à UFF foi recebida com a seguinte resposta “[...] a Superintendência de Tecnologia da Informação da UFF confirma a existência do acordo entre a UFF e a empresa Google. Contudo, em função de cláusulas contratuais, seu teor não poderá se divulgado sem o consentimento formal dos partícipes". Mesmo com pedido de reconsideração acatado pela Ouvidoria Geral da União, não houve seguimento ao processo.

${ }^{2}$ Acrônimo que designa cinco das maiores empresas de tecnologia do mundo:: Google, Apple, Facebook, Amazon e Microsoft. ${ }^{3}$ A pergunta solicitou dados de ifmg.br. No entanto, não julgamos pertinente refazê-la.

$4 "[\ldots]$ to extol the consumer is to deny the citizen. When consumers begin to act, the fundamental decisions have already been made"

5“[...] the suggestion that if corporations simply declare what their privacy standards are, consumers could choose those that suit them, is erroneous if not misleading. The statements are written in legalese, in terms few can penetrate; the privacy settings provided are complex, cumbersome, and frequently revised - after the users have posted information on the site that they cannot erase."

\section{Referências}

ALIM, Frida et al. Spying on students: School-issued devices and student privacy. San Francisco: Eletronic Frontier Foundation, 2017. Disponível em: https://bit.ly/3kVXIBJ. Acesso em: 20 mar. 2020.

AMIEL, Tel. Conteúdos educacionais, abertura e vigilância na ecologia digital. In: ALMEIDA, Fernando et al. (ed.). Cultura, educação e tecnologias em debate. São Paulo: SESC, 2020. p. 58. 
AMIEL, T. et al. Os modos de adesão e a abrangência do capitalismo de vigilância na educação brasileira. Zenodo, 14 set. 2021. DOI: https://doi.org/10.5281/zenodo.5507265. Disponível em: <https://zenodo.org/record/5507265>. Acesso em: 14 set. 2021.

BRASIL [Constituição (1988)]. Constituição da República Federativa do Brasil. Diário Oficial da União: Brasília, DF, Seção 1, p. 1, 5 out. 1988. Disponível em: https://bit.ly/2VDBNGg. Acesso em: 20 mar. 2020.

BRASIL. Lei no 8.666, de 21 de junho de 1993. Regulamenta o art. 37, inciso XXI, da Constituição Federal, institui normas para licitações e contratos da Administração Pública e dá outras providências. 1993. Diário Oficial da União: Brasília, DF, Seção 1, p. 8269, 22 jun. 1993. Disponível em: https://bit.ly/3yHora1. Acesso em: 20 mar. 2020.

BRASIL. Decreto n ${ }^{\circ}$ 9.764, de 11 de abril de 2019. Dispõe sobre o recebimento de doações de bens móveis e de serviços de pessoas físicas ou jurídicas de direito privado pelos órgãos e pelas entidades da administração pública federal direta, autárquica e fundacional. Diário Oficial da União: Brasília, DF, Seção 1, p. 14, 11 abr. 2019. Disponível em: https://bit.ly/3yHora1. Acesso em: 20 mar. 2020.

CENTRO REGIONAL DE ESTUDOS PARA O DESENVOLVIMENTO DA SOCIEDADE DA INFORMAÇÃO - CETIC.BR. TIC Educação 2018. Brasília: NIC.br, 2019. Disponível em: https://bit.ly/38uR1Au. Acesso em: 30 mar. 2020.

COULDRY, Nick; MEJIAS, Ulisses A. Data colonialism: Rethinking big data's relation to the contemporary subject. Television \& New Media, [S. 1.], v. 20, n. 4, p. 336-349, 2019. DOI: https://doi.org/10.1177/1527476418796632. Disponível em: https://bit.ly/3jurf5G. Acesso em: 20 mar. 2020.

COUTINHO, Doigo R.; KIRA, Beatriz. Por que (e como) regular algoritmos? Jota, 2 maio 2019. Disponível em: https://bit.ly/3jtnduw. Acesso em: 20 mar. 2020.

CRUZ, Leonardo Ribeiro da; SARAIVA, Filipe de Oliveira; AMIEL, Tel. Coletando dados sobre o Capitalismo de Vigilância nas instituições públicas do ensino superior do Brasil. In: SIMPÓSIO INTERNACIONAL LAVITS, 6. 2019, Salvador. Anais [...]. Salvador: LAVITS, 2019. p. 1-18. Disponível em: https://bit.ly/3kC9HnH. Acesso em: 20 mar. 2020.

CRUZ, Leonardo Ribeiro da; VENTURINI, Jamila Rodrigues. Neoliberalismo e crise: o avanço silencioso do capitalismo de vigilância na educação brasileira durante a pandemia da Covid-19. Revista Brasileira de Informática na Educação, Porto Alegre, v. 28, p. 1060-1085, 15 dez. 2020. e-ISSN: 2317-6121. DOI: http://dx.doi.org/10.5753/rbie.2020.28.0.1060. Disponível em: https://bit.ly/3BqKW19. Acesso em: 20 mar. 2021.

ETZIONI, Amitai. The Privacy Merchants: What is to be done? University of Pennsylvania Journal of Constitutional Law, [S. 1.], v. 14, n. 4, p. 929, 1 mar. 2012. Disponível em: https://bit.ly/3zspo77. Acesso em: 20 mar. 2020.

EVANGELISTA, Rafael. Review of Zuboff's The Age of Surveillance Capitalism. Surveillance \& Society, v. 17, $\mathrm{n}$. 1/2, p. 246-251, 2019. Disponível em: https://bit.ly/3jtxgjd. Acesso em: 20 mar. 2020.

EVANGELISTA, Rafael; FIRMINO, Rodrigo José. Brazil. Modes of pandemic existence: territory, inequality, and technology. In: TAYLOR, Linnet et al. (ed.). Data Justice and COVID-19: Global Perspectives. London: Meatspace Press, 2020. p. 100-107.

FUCHS, Christian. Communication and Capitalism. Londres: University of Westminster Press, 2020.

KWET, Michael. Operation Phakisa Education: Why a Secret? Mass Surveillance, Inequality, and Race in South Africa's Emerging National e-Education System. First Monday, Illinois, 2 dez. 2017. DOI: https://doi.org/10.5210/fm.v22i12.8054. Disponível em: https://bit.ly/38uUDCy. Acesso em: 6 abr. 2021.

LIMA, S. Educação, Dados e Plataformas: Análise descritiva dos termos de uso dos serviços educacionais Google e Microsoft. São Paulo: Iniciativa Educação Aberta, ago. 2020. 
Disponível em: <https://doi.org/10.5281/zenodo.4012539>.

LINDH, Maria; NOLIN, Jan. Information we collect: Surveillance and privacy in the implementation of Google Apps for Education. European Educational Research Journal, [S. 1.], v. 15, n. 6, p. 644-663, 2016. Disponível em: https://bit.ly/3DAaNc8. Acesso em: 20 mar. 2020.

MOROZOV, Evgeny. To save everything, click here: The folly of technological solutionism. [S. 1.]: Public Affairs, 2013.

MOROZOV, Evgeny. Big Tech: a ascensão dos dados e a morte da política. São Paulo: Ubu, 2018.

PARRA, Henrique et al. Infraestruturas, economia e política informacional: O caso do Google Suite for education. Mediações, Londrina, v. 23, n. 1, p. 63-99, 2018. Disponível em: https://bit.ly/2WxbeDD. Acesso em: 20 mar. 2020.

SANTOS, Laymert Garcia dos. A informação após a virada cibernética. In: SANTOS, Laymert Garcia dos et al. Revolução tecnológica, internet e socialismo. São Paulo: Fundação Perseu Abramo, 2003. p. 9-33.

SILVEIRA, Sergio Amadeu; AVELINO, Rodolfo; SOUZA, Joyce. A privacidade e o mercado de dados pessoais. Liinc em Revista, Brasília, v. 12, n. 2, p. 217-230, nov. 2016. DOI: https://doi.org/10.18617/liinc.v12i2.902. Disponível em: https://bit.ly/3DAY7Sj. Acesso em: 24 nov. 2020.

SOLOVE, D. J. I've got nothing to hide and other misunderstandings of privacy. San Diego L. Rev., [S. 1.], v. 44, p. 745-772, 2007. Disponível em: https://bit.ly/3mIDbmy. Acesso em: 20 mar. 2020.

ZUBOFF, S. A era do capitalismo de vigilância: a luta por um futuro humano na nova fronteira do poder. Rio de Janeiro: Intrínseca, 2020. 